\title{
VIII.
}

\section{MiBbildungen bei den farbigen Bewohnern der deutschen Schutzgebiete}

\author{
Von \\ Dr. Gustar Heim \\ in Bonn.
}

Während eines Aufenthaltes in Lüderitzbucht (Deutsch-Südwestafrika) vertrat ich einen dortigen Kollegen drei Wochen in seiner Eingeborenenpraxis. Dabei machte ich mir Notizen über Formation der Ohrmuschel und der Zähne, deren Bildungsfehler ja in der Psychiatrie unter den körperlichen Entartungszeichen eine Rolle spielen. Ich gewann den Eindruck, daß hierin die Farbigen sich von den Europäern im allgemeinen nicht unterscheiden. Das angewachsene Ohrläppchen, welches den Kriminalpsychologen so bedeutungsvoll erscheint, fand ich häufig; dabei auch, und zwar unter 79 Farbigen sechsmal, den von Morel betonten Übergang desselben mittels langen spitzen Endes in die Wangenhaut ${ }^{1}$ ). In 10 Fällen war bei Farbigen verschiedenster Herkunft kein Ohrläppchen vorhanden, in anderen war es sehr klein, kaum ausgebildet oder nicht deutlich differenziert und nur bei wenigen vollkommen. Es handelte sich vorwiegend um aus dem Kapland stammende Farbige (Cape boys), angeblich grobenteils aus Ehen zwischen Buren und Negern hervorgegangen, ferner um Ovambos aus dem Norden DeutschSüdwestafrikas, Kamerunneger, Hereros und vereinzelte andere, fast sämtlich männlichen Geschlechts. Da meiner Erfahrung nach in Deutschland das weibliche Geschlecht ungemein häufig verbildete Ohrmuscheln zeigt, glaube ich, bei den eingeborenen Afrikanerinnen noch mehr als bei dem männlichen Teil jene Deformitäten erwarten zu dürfen.

Von der gerühmten Schönheit der Zähne der Farbigen konnte ich mich in Lüderitzbucht nicht überzeugen. Wenigstens war bei mehreren fast das ganze Gebiß durch Fäulnis zerstört, bei einigen erschienen sie verkümmert oder verschieden groß, z. B. die Schneidezähne auf der linken Seite viel länger als auf der rechten. Auch die von Talbot als Degenerationszeichen angeführte weite Stellung der Zähne ${ }^{2}$ ) (breite Lücken) sah ich wiederholt. Ein Teil hatte ein gutes und schönes Gebiß. Offenbar läßt der Kontrast mit der dunklen Haut dasselbe in erhöhtem Maße weiß und blendend sich abheben.

Behufs weiterer Information über diesen Gegenstand sah ich die bisher erschienenen Medizinalberichte über die deutschen Schutzgebiete, Jahrgänge 1903/04 bis einschließlich 1910/11, durch ${ }^{3}$ ). Ich fand, daß auch überzählige Zähne

1) Morel, Traité des dégénérescences de l'espèce humaine. Paris 1857.

2) Talbot, The degenerate jaws and teeth. Internat. Dent. Journ. 1897.

3) Erschienen in Berlin bei E. S. Mittler \& Sohn.

Virchows Arehiv f. pathol. Anat. Bd. 220. Hft. 2. 
bei den Farbigen vorkommen. In Lome (Togo) sind sie angeblich häufig und treten bisweilen in größerer Zahl meist im Oberkiefer auf. Auch auf der Station Nauru der Marshall-Inseln wurden sie beobachtet.

Dieser Befund darf nicht überraschen, da nämlich bei den Farbigen auch andere Körperteile in Überzahl gefunden wurden. Überzählige Finger scheinen ziemlich häufig zu sein. Im Bericht für 1909/10 schreibt man aus Lome in. Togo:

„,Ferner beobachtet man nicht selten überzählige Finger. Diese sind entweder kleine Hautwarzen an der Außenseite der Grundglieder beider kleiner Finger oder es schließt sich an die Warze ein kleines stecknadelkopfgroßes Knorpelstückchen an, von welchem ein dünner Sehnenfaden abgeht zur Strecksehne des kleinen Fingers. In einem Falle war mit dem Knorpelstückchen ein $7 \mathrm{~mm}$ langer und $2 \mathrm{~mm}$ dicker Knochen gelenkartig verbunden. Früher wurde an dieser typischen Stelle beiderseits ein vollständig ausgebildeter zweigliedriger Finger beobachtet in der Größe des kleinen Fingers eines zweijährigen Kindes."

Im gleichen Jahre sah man in Deutsch-Ostafrika bei einem 30 Jahre alten Manyemaweib an den Metakarpophalangeal-Gelenken der beiden kleinen Finger je einen sechsten knorpelig sich anfühhlenden, aus einem Gliede bestehenden Finger, deren jeder einen kleinen Nagel trug. Diese Glieder waren nicht gelenkig, die Füße normal. Auch bei einem Kinde fand man dort einen sechsten Finger, bei zwei anderen je sechs Finger und je sechs Zehen. In all diesen Fällen konnten bei den Angehörigen keine Mißbildungen festgestellt werden. Im Bezirk Bukoba (DeutschOstafrika) sollen viele solche Mibbildungen vorkommen. 1910/11 schreibt von dort Stabsarzt Dr. Grothusen:

„Überzählige sechste Finger sieht man unter den Farbigen der Kolonie häufig. Die Lokalisation dieser MiBbildung sah ich bisher nur am ulnaren Rande neben dem Kleinfinger. In Ndama (Kianja) sah ich jedoch einen Mann, der beiderseits wohl ansgebildete zweite Daumen hatte. Diese waren nur etwas kleiner wie die eigentlichen Daumen und am Mlittelgelenk gebeugt und ankylotisch fixiert. Aktiv konnten sie nicht bewegt werden."

Auch aus Neu-Langenburg (Deutsch-Ostafrika) wird 1910/11 von Geisler von einem Manne mit sechs Fingern berichtet. Der überzählige bestand beiderseits nur aus dem Nagelglied mit gut entwiekeltem Nagel, welches an der Grundphalanx der kleinen Finger locker aufsaß.

Geisler sah damals dort noch andere Bildungsfehler der Extremitäten. Fin erwachsener Eingeborener hatte ganz auffallend kurze Oberarme, sonst war er normal entwickelt. Das Längenverhältnis der Ober- zu den Unterarmen erinnerte an die Anthropoiden, besonders die Gorillas. Ein anderer Eingeborener hatte normal entwickelten Oberkörper, dagegen stark verkürzte und verkrümmte Ober- und Unterschenkel und verkürzte Arme. Grothusen erzählt von einer alten Frau in Njatakara in der Landschaft Usambiro (Deutsch-Ostafrika), welche eine angeborene vollkommene Verkümmerung der rechten Hand hatte. Die fünf Finger waren nur als kleine Nagelgliedanhänge angedeutet.

Auch Plattfuß scheint nach verschiedenen Berichten bei den Farbigen unserer afrikanischen Schutzgebiete nicht selten zu sein. Spitzfuß wurde 1907/08 
im Bezirk Viktoria (Kamerun) dreimal, Pes valgus damals in Togo einmal bei Farbigen beobachtet. In Mpapua (Ostafrika) sah Stolowsky bei einem voll erwachsenen, etwa 30 Jahre alten Neger doppelseitigen Pes varus congenitus. Beide Füße standen in hochgradigster Supination; der Mann vermochte dabei aber sehr gewandt und schnell zu gehen. Noch andere Mißbildungen der Knochen und Gelenke fielen auf. Über Hasenscharten berichtet Grothusen aus Bukoba (Deutsch-Ostafrika):

,,Nachdem eine Hasenscharte bei einem 6 jährigen Kinde mit Erfolg operiert war, kamen verschiedene andere in Behandlung. Sie wurden alle nach Königscher Art operiert. Das Resultat war immer gut. Finmal mußte ein stark prominierender Zwischenkiefer entfernt werden " ${ }^{1}$ ).

Geisler fand unter den Wungus (Deutsch-Ostafrika) zwei erwachsene Frauen mit sehr starker, angeblich angeborener Kyphose der Lenden- und unteren Brustwirbelsäule. Die eine von ihnen hatte ein normal entwickeltes Kind.

Grothusen (Bukoba) sah in Deutsch-Ostafrika wiederholt Zwergwuchs. Zunächst war es ein etwa 40 jähriger Mann, der $120 \mathrm{~cm}$ groß war, und ein etwa 18 jähriges Mädchen, das knapp $100 \mathrm{~cm}$ maß. Die beiden Zwerge waren, abgesehen von einem großen Kropf, völlig wohl proportioniert gebaut und geistig normal. Beide stammten aus verschiedenen Familien. Ihre Eltern und Geschwister zeigten keine Abnormitäten. Einige weitere Zwerge gewahrte er in der Landschaft Lukorra (Ussuwi). Auch diese stammten aus angeblich ganz normal gebauten Familien ${ }^{2}$ ).

Phimose kommt durchgängig bei den Farbigen Afrikas und der Südsee vor. Im Bezirk Rabaul (Deutsch-Neu-Guinea) z. B. wurden 1910/11 unter 1546 farbigen Kranken 10 an diesem Fehler behandelt. Im Gouvernements-Krankenhaus in Tanga (Deutsch-Ostafírika) wurden damals von den 6759 dort (einschließlich Poliklinik) behandelten Farbigen 161 operiert. Dabei waren 23 Phimosen-Operationen.

Ungleich häufiger noch und nahezu aus allen deutschen Schutzgebieten werden Hernien gemeldet, ganz besonders aus Kamerun. Ungeheure Zahlen werden hier in verschiedenen Jahrgängen angeführt. 1910/11 kamen in Kamerun auf 702 kranke Europäer $2=0,28 \%$, auf 9688 kranke Farbige dagegen $265=2,74 \%$ Unterleibsbrüche, dabei 22 eingeklemmte. Im Text heißt es:

,Die Unterleibsbrüche kommen in immer steigender Zahl zur ärztlichen Behandlung. Der Neger, nament]ich der arbeitende, kann noeh weniger als der arbeitende Europäer ein Bruchband gebrauchen. Die Schmerzen, die häufigen jähen Todesfälle durch Einklemmung des Bruches und die kolossalen Dimensionen, welche einige dieser Brüche annehmen, lassen die Radikaloperation trotz mancher Mißerfolge als einen großen Fortschritt erscheinen."

In dem genannten Jahre wurden darum auch weitaus die meisten Fälle, nämlich 231, operiert. Es ist auffallend, daß der indolente und operationsscheue Farbige sich so bereitwillig der Radikalbehandlung dieses Gebrechens unterwirft. Aufklärung darüber gibt eine Notiz aus Duala in Kamerun (1908/09, S. 211):

1) Med.-Ber. 1910/11, S. 252.

2) Med.-Ber. 1910/11, S. 253. 
„Im ganzen wurden (im Hospital Duala) von Unterleibsbrüchen 128 behandelt. Von weit aus dem Hinterlande kommen die Kranken, um von ihrem Übel befreit zu werden, sehr häufig ohne veranlassende eigentliche Beschwerden. Der tiefere Grund ist ja bekanntlich der, daß die schwarze Damenwelt dieses Leiden für anstößig ansieht und den damit behafteten ihre Gunst zu versagen pflegt."

Nabelbrüche gelten dagegen als Schönheitszeichen! Wenigstens ist dies angeblich in dem Bezirk Lomie in Kamerun der Fall, wo Unterleibsbrüche sehr selten sein sollen. Es scheint deren Verbreitung in Kamerun sehr verschieden zu sein. Ein Urteil darüber aus Garua lautet:

„Leistenbrüche wurden weniger wie an der Küste gesehen (nur drei). Auch die Ärzte von dem Hochlande in Dschang und Bamenda melden, daßB ihnen im Gegensatz zur Küste dort fast gar keine Leistenbrüche vorkämen. Bei der Ähnlichkeit der Bevölkerung und der Ernährung ist das auffallend, $O \mathrm{~b}$ speziell die Häufigkeit der Leistenbrüche bei der Küstenbevölkerung Beziehung zu dem Rudern hat, müBte noch weiter untersucht werden" (1909/10, S. 321).

Beiläufig bemerkt ist auch Hy dr o zele bei den Farbigen der deutschen Schutzgebiete sehr verbreitet. Eine Meningomyelozele wurde bei einem Banyanenkind in Daressalam operiert ${ }^{1}$ ). An Varikozele litt ein Farbiger in Togo.

Zwei Fälle von Monorchidie fand ich in einer Krankenliste der Farbigen Anechos (Kamerun) und einen Fall von Hypospadie in einer solchen Tabelle von Samoa. Geisler sah in Neu-Langenburg in Deutsch-Ostaffrika bei zwei Askariboys angeblich angeborenes Fehlen beider Hoden sowie der Prostata. Der eine von ihnen war im Verhältnis zu seinem geschätzten Alter im Längenwachstum zurückgeblieben und zeigte eine ziemlich starke allgemeine Fettentwicklung.

In Lome (Togo) fand man angeborene Atresie der mittleren Hälfte der Scheide mit einer über faustgroßen derben Geschwulst (Hämatosalpinx) links vom Uterus bei einem sonst normal entwickelten Negermädchen von etwa 17 Jahren. Einem Berichterstatter von Lomie in Kamerun (1908/09) sind von anatomischen Eigentümlichkeiten der dortigen Eingeborenen aufgefallen erhebliche Größenunterschiede zwischen den beiden Nieren, insofern die linke Niere stets größer war als die rechte, sowie bei einzelnen eine ganz bedeutende Verlängerung des Dickdarms. Letztere führt er allerdings auf die sehr voluminöse Nahrung zurück. In Mahenge in Deutsch-Ostafrika fand man bei der Laparatomie bei einem etwa 28 jährigen Mnyamwesi keine Spur von Wurmfortsatz.

Im Anschluß an einen Fall von Fistula auris congenita wird 1908/09 im Bericht aus Lome (Togo) bemerkt:

„Diese Fisteln, meist beiderseitig, sind sehr verbreitet, werden selten Gegenstand ärztlicher Behandlung, wenn das sezernierte, milchige Sekret übelriechend wird."

Hemeralopie und Nyktalopie fand ich einigemal bei Farbigen Kameruns und der Südsee erwähnt. 1909/10 wurden auf Nauru (Marshall-Inseln) von 4000 kranken Farbigen sogar 32 an Hemeralopie behandelt. Wick in Rabaul (Deutsch-

1) Ausführliche Beschreibung in Med.-Ber. 1910/11, S. 253. 
Neu-Guinea) fand unter den Eingeborenen erstaunlich viel Leute mit höheren Graden von Hyperopie und Myopie und als deren Folge Strabismus.

Sicherlich stellen die hier von mir angeführten Mißbildungen nur einen kleinen Teil der während der genannten Zeit wirklich vorhandenen vor. Zunächst begibt sich der sehr indolente Farbige wegen solcher Dinge nicht leicht zum Arzte. Sodann sind unsere Schutzgebiete noch sehr schwach mit Ärzten besetzt, so daß auch darum weitaus die meisten Krankheiten und Abnormitäten der Beobachtung entgehen. In Ostafrika z. B., welches doppelt so groß wie Deutschland ist, sind nicht viel mehr als 40 Ärzte. Die Verbildeten und Verkrüppelten kommen auch wohl in geringerer Zahl zu den Arbeitszentren, wo die Ärzte stationiert sind. Mit solehen Fehlern geborene Kinder werden zudem bei vielen Stämmen frühzeitig aus der Welt geschafft.

Nach alledem haben wir keinen Grund zu der Annahme, daß hinsichtlich der Mißbildungen die Farbigen der deutschen Schutzgebiete im allgemeinen anders gestellt seien, als die Europäer.

\title{
IX. \\ Die Histologie der Blutungen und die extra- und intra- vaskuläre Thrombose.
}

\author{
Von \\ Professor Dr. Hugo Ribbert in Bonn. \\ (Hierzu 9 Textfiguren.)
}

Bei Untersuchung verschiedener Hämorrhagien fiel es mir wiederholt auf, daß große Abschnitte des ergossenen Blutes, abgesehen von den darin eingeschlossenen zelligen und sonstigen Bestandteilen des von ihnen durchsetzten Gewebes, nur aus roten Blutkörperchen bestanden, daß die weißen Elemente dagegen fehlten. Ich sah es u. a. in den subendokardialen Blutungen, die sich im Gebiete des Stammes des linksseitigen Reizleitungssystems nicht selten finden und die ich in einem eigenen Aufsatz ${ }^{1}$ ) besprochen habe. Hier konnte ich innerhalb der Blutmassen, durch die das Gewebe der Bündel- und der angrenzenden Myokardfasern auseinandergedrängt wird, in großen Bezirken keine Leukozyten nachweisen oder sie waren doch nur hier und da in vereinzelten Exemplaren vorhanden. Das fiel besonders dann auf, wenn durch das ausgetretene Blut eine gefiullte Vene hindurchzog. Man sah in ihr die gewöhnliche Anzahl weißer Blutzellen, während sie außen voll-

1) Wien. med. Wsehr. 1915. 